\title{
Transthoracic image guided biopsy
}

\author{
Robert Dick
}

Department of Radiology, The Royal Free Hospital, Hampstead, London NW3 2QG, UK.

\begin{abstract}
Summary: Transthoracic image guided biopsy is now firmly established in the armamentarium of diagnostic tests to establish the nature of a mass in the lung, pleura, mediastinum or chest wall. It may be performed under computed tomographic, ultrasonic or fluoroscopic control, and provided a fine needle technique is employed, morbidity is low. Worldwide reports show a $90 \%$ true positive yield and a false positive rate of less than $1 \%$.
\end{abstract}

\section{Introduction}

The discovery of a discrete pulmonary lesion on the chest radiograph demands a precise and rapid diagnosis in view of the ever-present risk of malignancy. Biopsy may be undertaken by means of the transbronchial route using the fibreoptic bronchoscope, via thoracotomy, or via a percutaneous transthoracic aimed approach using a wide choice of aspirating or cutting needles. One of these methods is usually invoked since sputum cytology and culture are relatively ineffective for obtaining a tissue diagnosis from this type of mass, particularly if it is situated in the outer two-thirds of the lung.

Percutaneous transpleural needle biopsy of the lung is not a new procedure. It has been practised over many years since its introduction in the last century by Leyden, who aspirated organisms causing a pneumonia ${ }^{1}$ and then by Menetrier, who three years later diagnosed a bronchial carcinoma by needle aspiration. ${ }^{2}$ Subsequently, several large series have been reported, using either an aspirating or a cutting technique..$^{3-5}$ Until recent years, percutaneous lung biopsy has not been as popular a procedure in the United Kingdom as in the United States and Scandinavia because of its complications. However, with the advent of aspiration biopsy using relatively narrow gauge needles, the procedure has become safer and now has a secure place in the diagnosis of isolated pulmonary lesions, many of which will be neoplastic. The biopsy is simply and rapidly performed, inexpensive, well tolerated by the patient, low in complications and readily repeated if necessary.

The incidence of a solitary pulmonary lesion on a chest radiograph is approximately $0.1 \% .^{6}$ Since both benign lesions, such as granulomata, and malignant ones, including metastases and primary adenocarcinomas, are peripheral, bronchoscopic biopsy is not possible. To establish the benign

Correspondence: R. Dick, F.R.A.C.R., F.R.C.R. nature of such a lesion saves the patient having a thoracotomy, whilst it is equally important to make the diagnosis of malignancy in a patient unfit or unsuitable for thoracotomy.

Of all solitary mass lesions seen on radiography in patients over 50 years, $30-50 \%$ will be carcinomata. ${ }^{7}$ Ethnic and geographical background, age, industrial exposure and smoking habits may suggest the nature of a lesion, but cannot allow a watertight diagnosis without tissues being obtained. A mass with localized rib destruction is likely to be malignant in nature, though not all nodules conz taining calcification will be benign. Granulomata and localized area of infection account for the majority of the non-malignant cases. ${ }^{8}$

\section{Indications}

The investigation of a mass lesion demonstrated on the chest radiograph, especially where it is peripherally situated and where sputum culture/cytology and bronchoscopy (if indicated) have failed to furnish a diagnosis (Figure 1).

The investigation of a mass lesion demonstrated on the chest radiograph in a patient known to be suffering from malignancy. Biopsy will establish whether or not the new lesion represents a metastasis or other pathology. Resection of a solitary pulmonary metastasis may significantly improve the patient's prognosis.

The investigation of an unresolved pneumonic consolidation, where the underlying cause (infective or non-infective) remains unknown despite other diagnostic tests. Sometimes direct retrieval of an offending organism is obtained, allowing specific chemotherapy to be utilised.

Any other persistent localized lung lesion that cannot be diagnosed by other methods.

Pleural masses, sometimes associated with rib destruction, though more often without it.

(C) The Fellowship of Postgraduate Medicine, 1988 

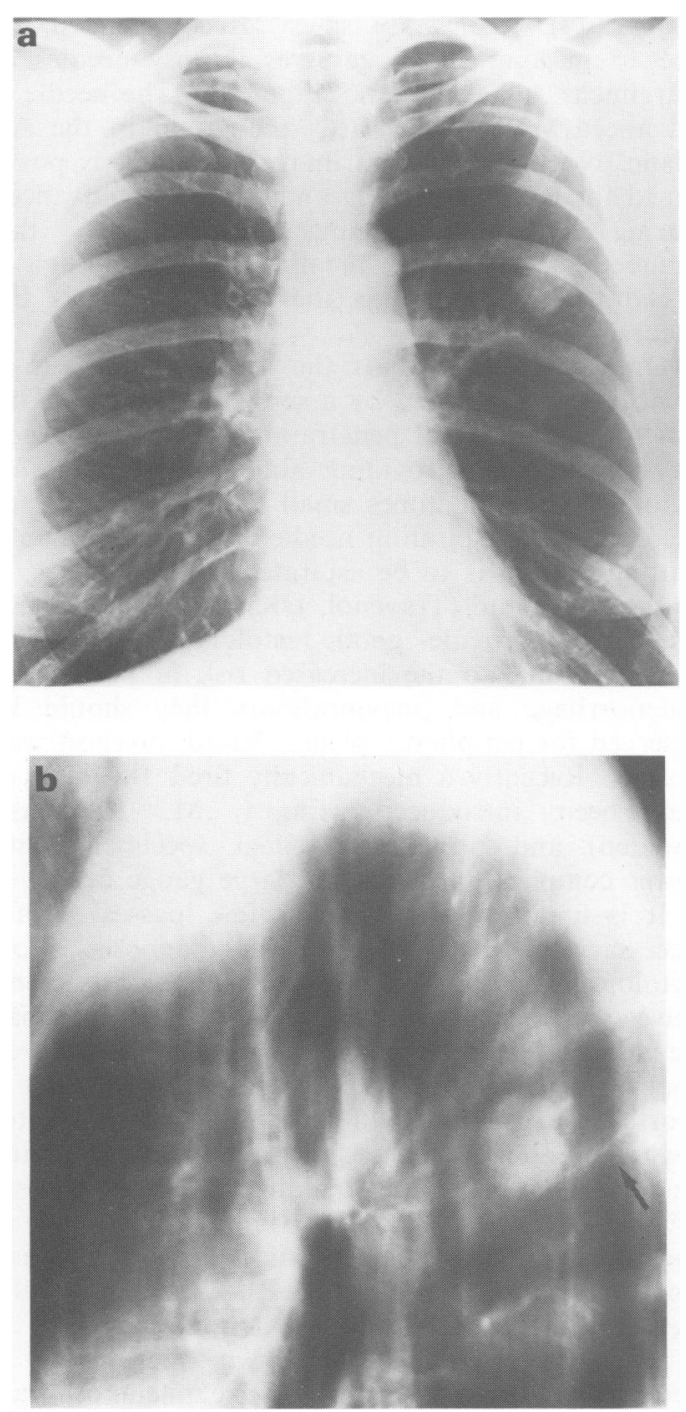

Figure 1 Sixty-four year old female, asymptomatic. (a) PA chest X-ray. (b) Lateral tomogram showing lesion of left upper lobe just above the posterior end of oblique fissure (arrowed). Biopsy showed squamous carcinoma.

\section{Contraindications}

These are few, and include: bleeding diatheses, including patients on anticoagulent therapy. In practice, a fine gauge $(21,22)$ needle can be safely employed in this group; suspected vascular lesions, such as an aneurysm or arteriovenous malformation; suspected echinococcus cyst; dissemination of hydatid may occur following needle puncture, and severe anaphylaxis result. This is the only absolute contraindication.

The following are theoretical contraindications, and provided small gauge needles are used and complications dealt with immediately, many in the following groups may safely be biopsied; severe pulmonary hypertension, which increases the risk of post-biopsy haemorrhage; contralateral pneumonectomy; severe pulmonary emphysema where lung function tests indicate that even a small pneumothorax would seriously impair the patient's respiratory reserve; the inability of the patient to cooperate with procedure, or his or her refusal to undergo definitive treatment irrespective of the biopsy result.

\section{Technique}

In order to achieve the best possible patient cooperation, biopsy is performed under local anaesthesia without premedication. A thorough explanation of the procedure should be given, well before the event, to the patient who ought to be able to lie flat on an X-ray table for 10-15 minutes without distressing dyspnoea.

Either computed tomography (CT) or fluoroscopy guidance can be used. The latter is less timeconsuming and cumbersome and allows a 'realtime' approach. Biplane fluoroscopy of a ' $\mathrm{C}$ ' arm (Figure 2) is ideal though not mandatory, and certainly not essential for large lesions. CT is more appropriate for lesions that are invisible on a PA chest radiograph (or in those where a small lesion present on the chest radiograph cannot be identified and therefore cannot accurately be biopsied). Difficult areas to biopsy include lesions behind the sternum, related to chest wall (Figure 3 ) or in the posterior costophrenic angle. It may also be used for lesions that are relatively inaccessible, i.e., adjacent to the scapula. Pleural lesions may be localized and biopsied using ultrasound or fluoroscopy $^{9}$ (Figure 4).

In all patients, a radiograph should be taken on the day of the biopsy prior to the procedure to ensure that the lesion has not altered (or cleared) since the film performed days or weeks before. ${ }^{10} \mathrm{~A}$ pre-requisite for successful biopsy is the accurate measurement of the position of the lesion and its distance from the nearest skin surface. This distance is calculated from PA and lateral chest radiographs, plus, when necessary, either conventional or computed tomography (Figure 1b).

Prior to biopsy the patient is placed in either the prone or supine position (lateral or oblique being uncomfortable) and 10-20 mg diazepam (emulsified diazepam, Kabivitrum Ltd. UK) is given intra- 


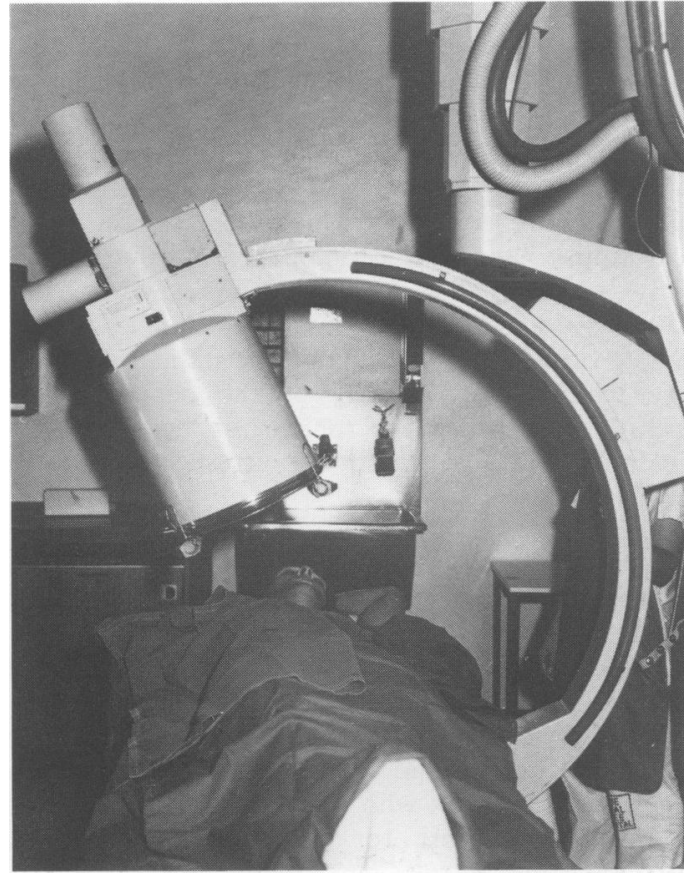

Figure 2 Patient on table prior to biopsy. The equipment, including the rotating $\mathrm{C}$ arm, is demonstrated.

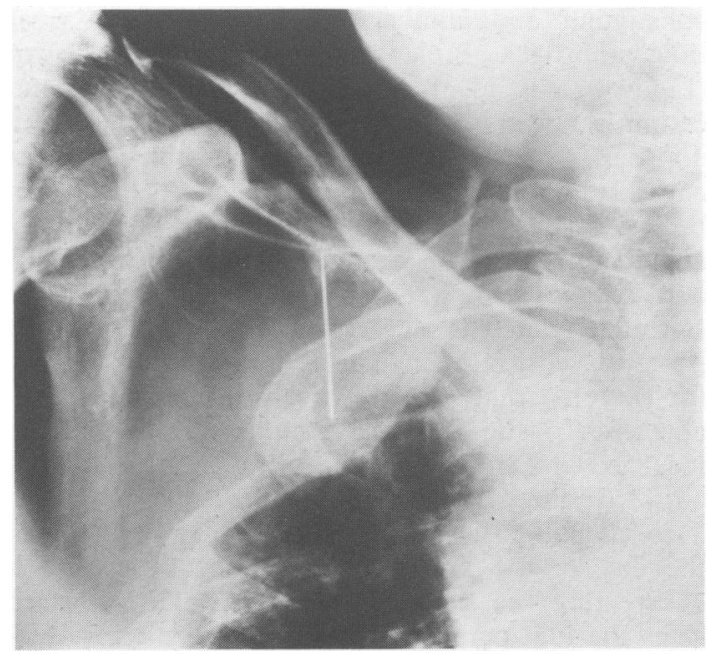

Figure 3 Note biopsy of a peripheral mass destroying the right second rib in this 50 year old male. Access from the back blocked by scapula. With arm abducted and clavicle elevated, an anterior approach becomes practicable. Biopsy showed undifferentiated carcinoma.

venously. Under fluoroscopy, a skin marker is placed over the lesion to be biopsied. Skin, chest wall and pleura are infiltrated with $5 \mathrm{ml} 1 \%$ lignocaine, and a needle selected for biopsy. Fine gauge
(20-22 FG) needles are safest. Recommended are the $15 \mathrm{~cm}$ long 20 gauge screw biopsy needles of Surgimed, A/S, Denmark (Figure 5). The needle is $\stackrel{\varnothing}{\varrho}$ advanced vertically towards the lesion in the AP plane. Next, the ' $C$ ' arm image intensifier is positioned for lateral screening and the needle advanced during lateral screening. Throughout this manoeuvre the patient breathes quietly, though is asked to breath-hold as the needle traverses the pleura.

Once the needle abuts the lesion, the stilette is क removed and replaced by a screw attachment. This has the advantage of penetrating hard lesions (such as granulomata) providing abundant material for cytology and sometimes small tissue fragments for histology. An aspirating needle is also safer than a cutting if fluid is to be aspirated from a cavity.

14FG 'Trucut' (Travenol, UK) needles have been used, and provide good histological specimens. However, due to the increased risk of pulmonary haemorrhage and pneumothorax they should be reserved for peripheral, pleural based, or chest wall lesions. Recently a mechanically fired $18 \mathrm{G}$ Trucut has been introduced (Biopty M, Radiplast, Sweden) and provides excellent specimens with fewer complications than the large gauge needle.

It is important to make as few 'passes' as are $\overrightarrow{\mathscr{O}}$ necessary to make a confident diagnosis. Mosi cytologists are happy to attend the biopsy them selves or to send a cytotechnician so that slides max be smeared correctly and the radiologist assured that material appears to be present (Figure 6). It is worthwhile waiting 15 minutes for the final cytological opinion to be rung through before removing the needle (or perhaps making a further biopsy) (Figure 7). Certainly up to 3 passes may be made at any one sitting, particularly if the lesion is small and/or relatively inaccessible. Passes should ideally be at different sites within the lesion.

After the needle is withdrawn, the patient is screened in expiration for possible pneumothorax. Irrespective of its presence or absence, the postprocedural advice is for the patient to lie laterally for two hours, with the side of the biopsy lowermost, as this may help to seal any small air leak. After four hours a chest radiograph is taken on expiration. If a pneumothorax is not present, the patient may be discharged home. However, the of presence of even a small pneumothorax means that the patient must remain in hospital overnight.

\section{Complications}

Pneumothorax is the commonest, and occurs in 15$25 \%$ of patients, of which approximately one in six require intercostal tube drainage. ${ }^{11}$ The percentage

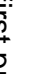
. 


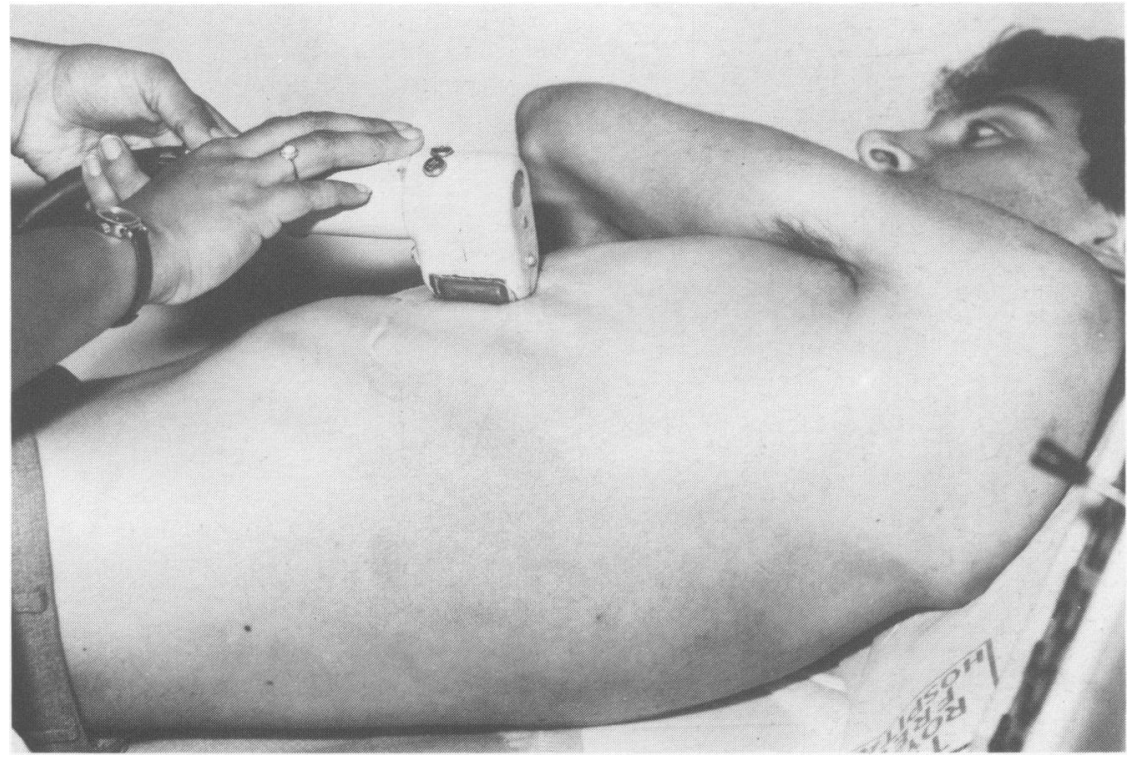

Figure 4 Ultrasound of pleura is being performed.

2.

\section{b}

Figure 5 (a) The needle and stilette used for aspiration biopsy. (b) Close-up view of spiralled tip of replacement stilette. 


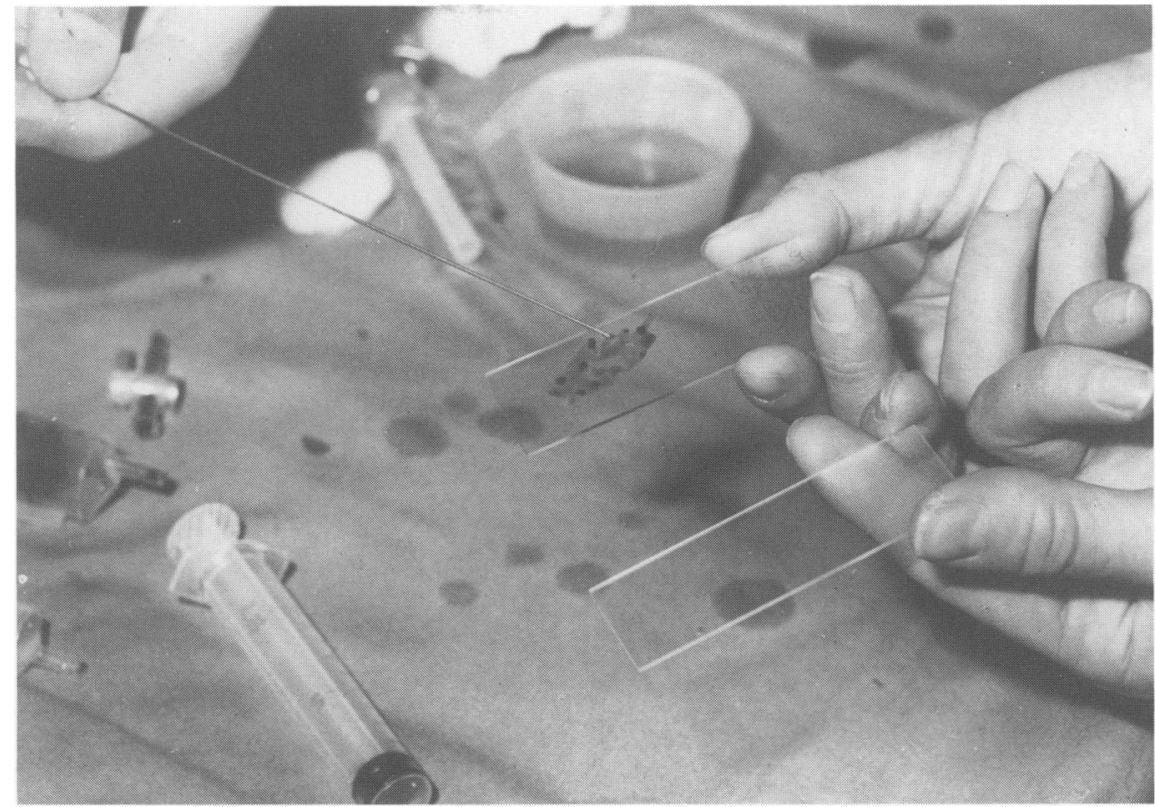

Figure 6 Ejecting material onto the slide surface, it will then be quickly spread into a thin film using a cover slip.
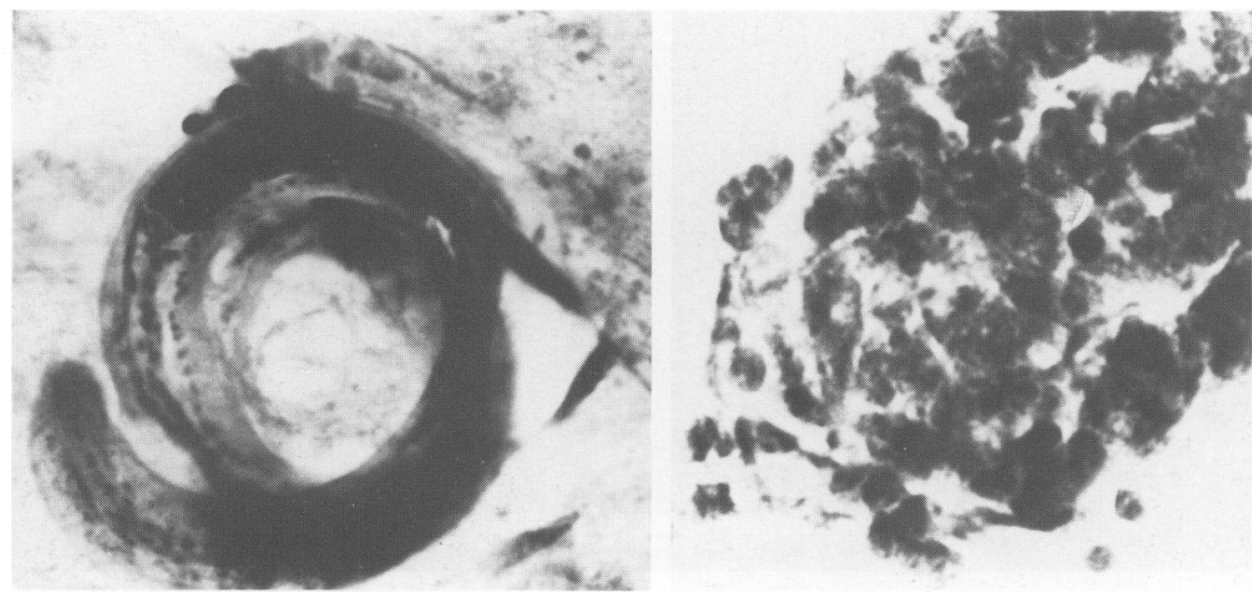

Figure 7 Aspiration cytology of a lung mass in a 55 year old West Indian male with peripheral lung shadow. Aspiration cytology revealed two pathologies within one FNAB specimen: (a) Strongyloides stercoralis larva (magnification $\times 250$ ); (b) Anaplastic small cell carcinoma (oat cell), magnification $\times 400$. (Courtesy of $\operatorname{Dr} \mathrm{A}$. Deery).

is based upon the visualization of a pneumothorax on a post-biopsy chest film, and the incidence is almost certainly very much higher, since supine CT scans performed after a biopsy invariably show a small anterior pneumothorax (Allison, personal communication, 1987). It is emphasized, however, that the majority of pneumothoraces are small, cause no respiratory embarrassment (though shoulder tip pain is common), and resorb spontaneously. Severe shoulder tip pain is usually $a \stackrel{\odot}{\triangle}$ warning of the presence of a moderate-sized pneu- $\stackrel{?}{?}$ mothorax. This apart, chest pain is not to be 7 expected after biopsy, though it has been reported $\overline{0}$ in a patient having a biopsy of a neurofibroma. ${ }^{10} \stackrel{\mathbb{D}}{\circ}$

The risk of pneumothorax is higher in patients $\frac{\overrightarrow{\mathbb{D}}}{\mathrm{Q}}$ with emphysema and in the elderly. Any radiologist 
performing lung biopsies must be able to deal with a large pneumothorax 'on site', since a 'tension' pneumothorax requires immediate tube drainage. In an emergency an FG8 Teflon catheter with side holes can be effectively inserted either at the biopsy site, the second anterior intercostal space, or high in the axilla on the affected side. The catheter assembly is attached to a Heimlich flutter valve to permit unidirectional airflow.

A small amount of pulmonary haemorrhage is not uncommon, and evidenced by a 'blush' of the lesion seen on screening after biopsy. A few patients will have a minor haemoptysis. They should be warned of this before the procedure, as haemoptysis is alarming to the unsuspecting patient. Severe haemoptysis is the most feared complication as it may cause death. It is extremely rare and more likely with large gauge cutting needles, lesions near the major pulmonary artery branches, in those with pulmonary hypertension, and in the elderly. Treatment is immediate bronchoscopy. In recent large series there have been no recorded fatalities from haemorrhage.

\section{Discussion}

A successful diagnostic procedure should have high patient acceptability, be simple and safe to perform, with a high positive yield and no false positive results: percutaneous lung biopsy fulfils these criteria, though its utility can ultimately only be judged on whether the result subsequently alters the patient's management.

Before the advent of image intensifiers, true positive rates as low as $40 \%$ were published. ${ }^{12}$ Modern screening facilities allow lesions as small as $1-2 \mathrm{~cm}$ to be seen and biopsied, some workers being confident enough to attempt those as small as $3 \mathrm{~mm}$ (Nordenstrom, personal communication). Results for USA and UK centres totalling fewer than 1,000 biopsies over an eight year period show a $90 \%$ true positive yield. ${ }^{4,10,13}$ Furthermore, the likelihood of mistakenly diagnosing a benign lesion as malignant (the false positive rate) is extremely low (well under $1 \%)$. The false negative rate in series is around $10 \% .{ }^{14}$

The site and depth of a lesion do not influence its suitability for biopsy, but inadequate specimens are more likely to be obtained from small deep lesions, especially by the inexperienced operator. Other reasons for the $10 \%$ false negative rate include necrosis, or infection around the tumour, or errors in cytology interpretation.

It is axiomatic that good cyto-pathological backup must be available before transthoracic biopsy is attempted. I have seen co-existent squa- mous cell carcinoma and myeloma correctly identified in smears from the same mass lesions, and recently Kelly et al. ${ }^{15}$ have reported small cell and squamous cell lung carcinomas developing sequentially at a single site. Should a negative biopsy result from one that clinically is felt to have a high likelihood of being malignant, then repeat biopsy is recommended. If this again is negative for malignant cells, it is extremely unlikely that the patient has a malignant lesion (Timmis, personal communication, 1987).

A challenge occurs with benign masses whose suspected innocence may be difficult to confirm on aspiration biopsy. A small firm nodule may move away from an advancing needle point, or be so hard as to defy puncture (Figure 8). Again, an
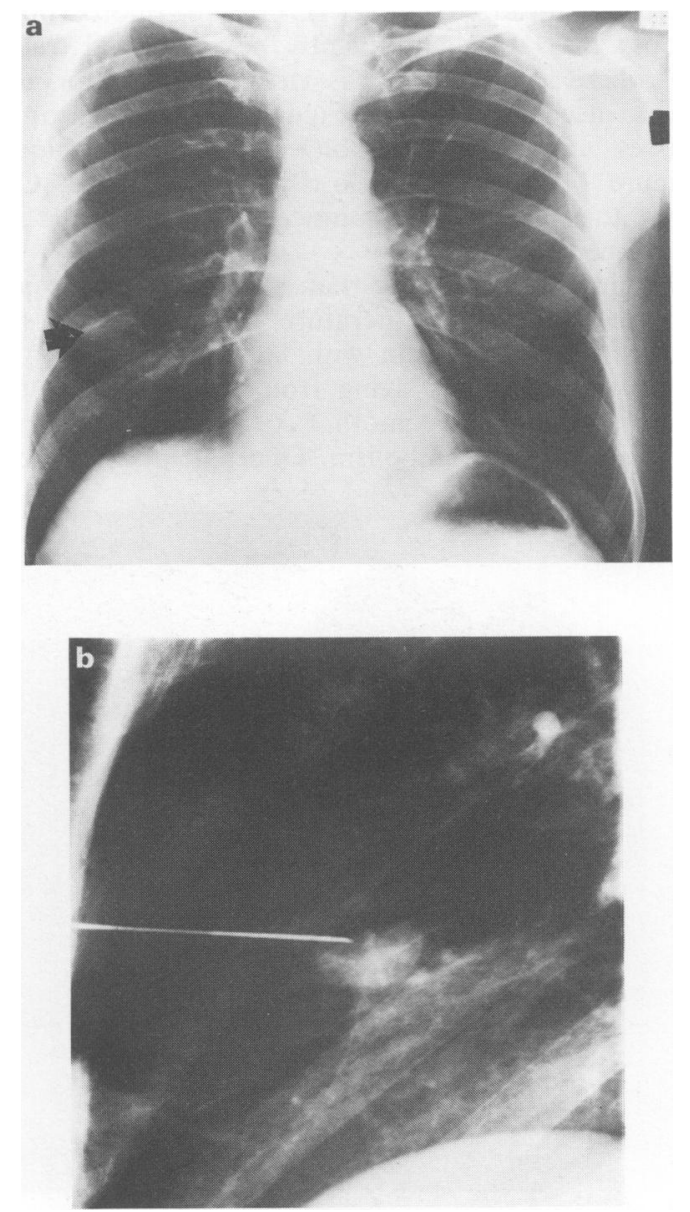

Figure 8 Fifty-five year old male with cerebral lymphoma. (a) PA chest X-ray showing small lesion right middle lobe (arrowed). (b) Lesion transfixed by aspiration biopsy. Cytology showed granuloma. 
inflammatory cellular aspirate may be in fact from an area distal to a neoplasm occluding a bronchus. It must be remembered that once a lesion has cavitated, biopsy should be from the periphery of the lesion rather than from the unrepresentative necrotic centre. It is disappointing that some benign pulmonary lesions that are common and mimic a carcinoma do not allow a confident or specific diagnosis on biopsy. These include chronic abcess, organizing pneumonia and pulmonary infection. Localized infection such as tuberculosis, staphylococcal disease and Aspergillus should be accurately diagnosed by an experienced interventional radiologist and his microbiological colleague.

In some patients tuberculosis and carcinoma may co-exist (Figure 9).

Chest wall lesions may be better visualized (and therefore localized) under CT than fluoroscopy. Biopsy may then conveniently be performed using CT where a fast scanner is virtually mandatory to check needle position (Figure 10). Mediastinal masses are usually suitable for either technique (Figure 11), though if close to great vessels, CT gives the biopsier reassurance that his needle is positioned safely.

From a total of more than 9,000 needle biopsies reported in the world literature, there have been six deaths. The association with large bore needles is clear, three patients dying from haemoptysis, one from tension pneumothorax (untreated) and another from air embolism. One patient who died

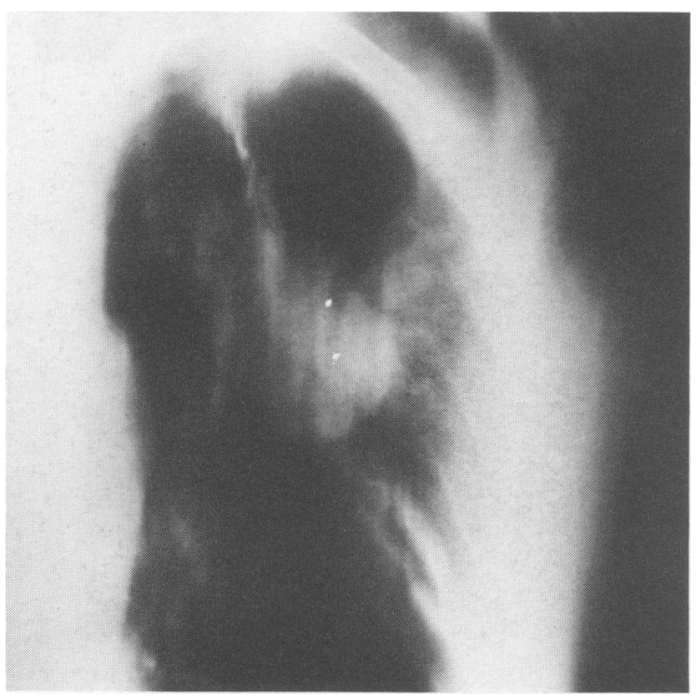

Figure 9 Seventy-two year old Greek male. Tomography of left upper lobe shows an irregular mass and also pleural calcification from known tuberculous pleurisy. Biopsy of mass showed squamous cell carcinoma.

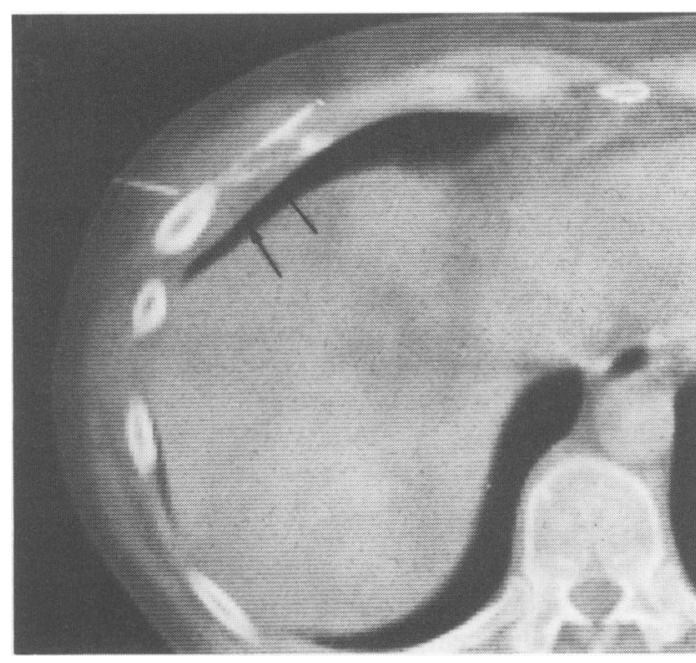

Figure 10 Known Hodgkin's disease, presenting with pain over right thoracic cage. Computed tomogram, and biopsy which confirmed Hodgkin's.

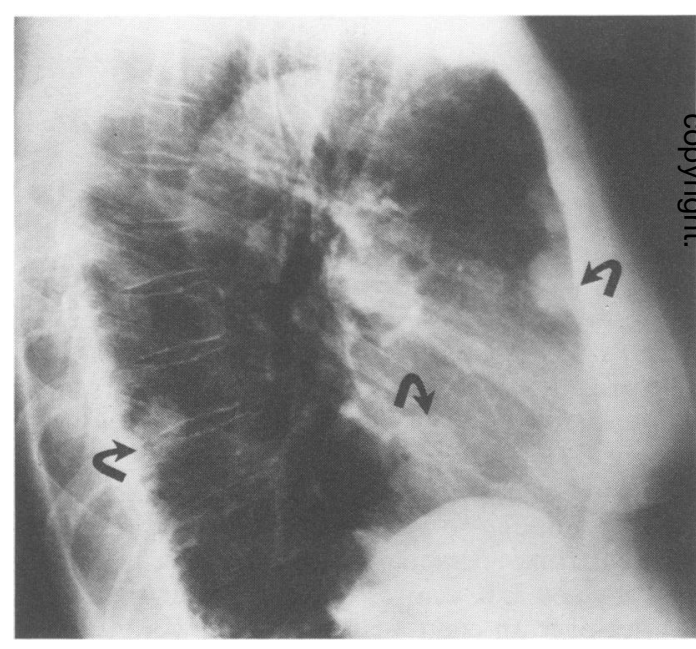

Figure 11 Fifty-eight year old female with previous removal of mediastinal thymoma. Lateral chest X-ray shows multiple pleural masses over the anterior, posterior and inferior surfaces (curved arrows). Biopsy of the anterior mass confirmed malignant thymoma.

from haemoptysis was so heavily sedated that he was unable to clear his airway. Clearly the radiolo- $\omega$ gist or chest clinician performing the biopsy must be ready to perform urgent intubation if required. A second case of fatal haemorrhage occurred in the $\mathbb{D}$ presence of severe pulmonary hypertension. With ${ }^{+}$ present use of fine needles, air embolism should not $\frac{T}{T}$ be feasible. The risk of needle track implantation of $\frac{\vec{D}}{\mathbb{D}}$ 
tumour cells remain low, Nordenstrom \& Bjork ${ }^{16}$ reporting only one proven case in a series of 4,000 biopsies.

A discussion of bronchoscopic biopsy will not be undertaken here but for masses close to the hilum this should be considered first. It is sensible to perform bronchoscopy on the fluoroscopy table, proceeding to percutaneous biopsy at the same session if the lesion is not visualized via the bronchoscope. ${ }^{17}$

\section{Conclusion}

Percutaneous needle biopsy within the thorax is a non-complex inexpensive 15 minute procedure which is tolerated well. ${ }^{18}$ Discomfort is minimal, some patients expressing surprise when a straightforward biopsy is pronounced completed. When

\section{References}

1. Leyden, H. Uber infectiose Pneumonie. Dtsch Med Wochenschr 1883, 9: 52-54.

2. Menetrier, P. Cancer primitif du Pulmon. Bull Soc Anatomy 1886, II: 643.

3. Dahlgren, S.E. \& Lind, B. Transthoracic needle biopsy or bronchoscopic biopsy? Scand J Resp Dis 1969, 50: 265-272.

4. Allison, D.G. \& Hemingway, A.P. Percutaneous needle biopsy of the lung. Br Med J 1981, 288: 875-878.

5. Gallo Curcio, C., Rinaldi, M., Tonachella, R. \& Donnorso, R.P. Role of percutaneous fine needle aspiration in the diagnosis of lung cancer. Our experience with 140 patients. Oncology 1983, 40: 177-188.

6. Good, C.A. The solitary pulmonary nodule: a problem of management. Radiol Clin N Am 1963, 1: 429-438.

7. Higgins, G.A., Shields, T.W. \& Keehn, R.J. The solitary pulmonary nodule. Arch Surg 1975, 110: $570-575$.

8. Sinner, W.N. Fine-needle biopsy of hamartomas of the lung. Am J Roentgenol 1982, 138: 65-69.

9. Flower, C.D.R. Percutaneous lung biopsy. Radiology Now 1987, 4: 23-25.

10. Dick, R. \& Timmis, B. Percutaneous needle biopsy of pulmonary tumours. In: Bates, M. (ed) Bronchial Carcinoma. Springer-Verlag, Berlin, 1984, pp 84-95.

11. Flower, C.B.R. Percutaneous lung biopsy, In: Whitehouse, G.H. \& Worthington, B.S. (eds) Techniques in Diagnostic Radiology. Blackwell Scientific Publications, Oxford, 1983, pp. 201-211. considering the risks of fine needle aspiration biopsy, and balancing these against the high diagnostic yield, it must be remembered that the only practical alternative at that particular stage in a patient's work up that includes negative bronchoscopy is thoracotomy, which has a higher complication rate than other biopsy methods. ${ }^{19}$ Although the accuracy of lung biopsy is over $90 \%$, it is stressed that not all conditions can be diagnosed, such as a pulmonary infarction resembling a mass. Notwithstanding, transthoracic biopsy should be used early for any lung, mediastinal or chest wall lesion suspected to be malignant, particularly if that lesion persists and cannot be diagnosed by noninvasive means. Whatever an initial or repeat biopsy result, it must not be viewed in isolation but taken in conjunction with a variety of other factors, including clinical history and examination, blood tests and perhaps further imaging.

12. Lauby, V.W., Burnett, W.E., Rosemund, G.P. \& Tyson, R.R. Value and risk of biopsy of pulmonary lesions by needle aspiration. $J$ Thorac Cardiovasc Surg 1965, 49: 159-172.

13. Poe, R.H. \& Tobin, R.E. Sensitivity and specificity of needle biopsy in lung malignancy. Am Rev Resp Dis 1980, 122: 725-729.

14. Berquist, T.H., Bailey, P.B., Cortes, D.A. \& Miller, W.E. Transthoracic needle biopsy. Mayo Clin Proc 1980, 55: 475-481.

15. Kelly, C.P., O'Donnell, D., West, B., Gallagher, L. \& Clancy, L. Small cell and squamous cell lung carcinomas: sequential occurrence at a single site. Thorax 1987, 42: 821.

16. Nordenstrom, B. \& Bjork, V.O. Dissemination of cancer cells by needle biopsy of the lung, $J$ Thorac Cardiovasc Surg 1973, 65: 671.

17. Johnson, N. McI., Dick, R., Casselden, P. \& Clarke, S.W. Fibreoptic bronchoscopy and fluoroscopic percutaneous needle biopsy - a combined approach to the investigation of thoracic lesions. Radiography 1979, 40: 273-275.

18. Gobien, R.P., Bouchard, E.A., Gobien, B.S., Valicenti, J.F. \& Vujic, I.V. Thin needle aspiration biopsy of thoracic lesions. Impact on hospital charges and patterns of patient care. Radiology 1983, 148: 65-67.

19. Zavala, D.C. \& Bedell, G.N. Percutaneous lung biopsy with a cutting needle; analysis of 140 cases and comparison with other biopsy techniques. Am Rev Respir Dis 1972, 106: 186-193. 